Check for

Check for

Cite as

Nano-Micro Lett.

(2022) 14:63

Received: 18 December 2021

Accepted: 22 January 2022

Published online: 21 February 2022

(C) The Author(s) 2022

\section{Ni Flower/MXene-Melamine Foam Derived 3D Magnetic/Conductive Networks for Ultra-Efficient Microwave Absorption and Infrared Stealth}

\author{
Haoran Cheng ${ }^{1}$, Yamin Pan $^{1}$, Xin Wang ${ }^{2}$, Chuntai Liu ${ }^{1}$, Changyu Shen ${ }^{1}$,

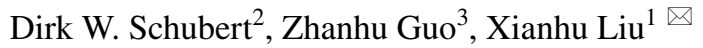

\title{
HIGHLIGHTS
}

- Ni-MXene/MF foam is synthesized via an electrostatic assembly and dip-coating process.

- The "micro-capacitor" structure of Ni/MXene and the 3D porous structure of MF endow the foam excellent impedance matching and wave absorption performance.

- The excellent heat insulation, infrared stealth, and flame-retardant performances are achieved.

\begin{abstract}
The development of multifunctional and efficient electromagnetic wave absorbing materials is a challenging research hotspot. Here, the magnetized Ni flower/MXene hybrids are successfully assembled on the surface of melamine foam (MF) through electrostatic self-assembly and dip-coating adsorption process, realizing the integration of microwave absorption, infrared stealth, and flame retardant. Remarkably, the Ni/ MXene-MF achieves a minimum reflection loss $\left(\mathrm{RL}_{\min }\right)$ of $-62.7 \mathrm{~dB}$ with a corresponding effective absorption bandwidth (EAB) of $6.24 \mathrm{GHz}$ at $2 \mathrm{~mm}$ and an EAB of $6.88 \mathrm{GHz}$ at $1.8 \mathrm{~mm}$. Strong electromagnetic wave absorption is attributed to the three-dimensional magnetic/conductive networks, which provided excellent

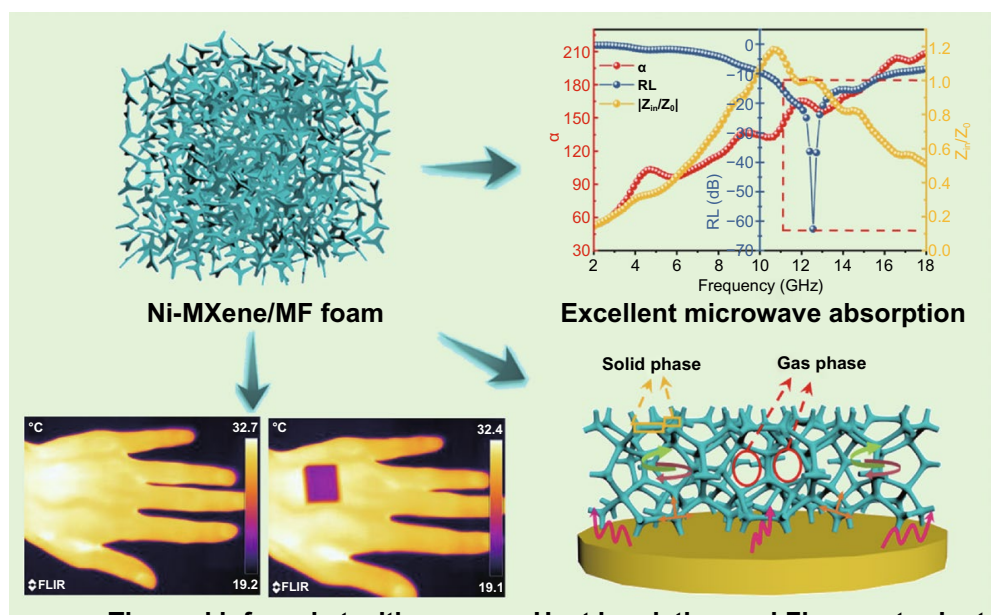

Thermal infrared stealth

Heat insulation and Flame-retardant impedance matching, dielectric loss, magnetic loss, interface polarization, and multiple attenuations. In addition, the Ni/MXene-MF endows low density, excellent heat insulation, infrared stealth, and flame-retardant functions. This work provided a new development strategy for the design of multifunctional and efficient electromagnetic wave absorbing materials.
\end{abstract}

KEYWORDS Ni-MXene/Melamine foam; Microwave absorption; Heat insulation; Infrared stealth; Flame-retardant

Haoran Cheng and Yamin Pan contributed equally to this work.

$\triangle$ Xianhu Liu, xianhu.liu@zzu.edu.cn

1 Key Laboratory of Advanced Material Processing \& Mold (Ministry of Education), National Engineering Research Center for Advanced Polymer Processing Technology, College of Materials Science and Engineering, Zhengzhou University, Zhengzhou 450002, People's Republic of China

2 Institute of Polymer Materials, Friedrich-Alexander-University Erlangen-Nuremberg, Martensstr. 7, 91058 Erlangen, Germany

3 Integrated Composites Laboratory (ICL), Department of Chemical \& Biomolecular Engineering, University of Tennessee, Knoxville, TN 37996, USA 


\section{Introduction}

The vigorous development of the $5 \mathrm{G}$ era, especially the development of next-generation electronic devices, network communications, and artificial intelligence, has provided high efficiency and convenience for our daily lives while bringing serious electromagnetic (EM) pollution [1-4]. Accordingly, the demand for EM wave absorbing materials has become increasingly urgent. Although there are many traditional EM waves absorbing materials, most of them do not meet the requirements of lightweight, thin matching thickness, wide effective absorption bandwidth (EAB), and strong absorption capacity [5-9]. Therefore, how to effectively absorb EM radiation and meet the requirements of EM wave absorbing materials has attracted widespread attention.

So far, graphene [10], $\mathrm{MoS}_{2}$ [11], CNT [12], SiC [13], and MXene have been recognized as representative candidate materials for high-efficiency EM absorbers. The twodimensional structure of MXene is considered to be the most potential absorbing material due to its strong conduction loss and polarization loss provided by excellent conductivity and abundant surface groups (-OH, -O, -F) [14]. In addition, MXene with a large specific surface area and aspect ratio could be used as an ideal platform carrier to couple with other magnetic components to solve the problem of impedance mismatch caused by its high conductivity [15]. Meanwhile, owing to the abundant functional groups, MXenes could combine with magnetic metal particles and effectively alleviate their stacking and agglomeration while enhancing impedance matching [16]. Liang et al. synthesized Ni/ MXene hybrid through a co-solvothermal method, which demonstrated a $\mathrm{RL}_{\min }$ of $-52.6 \mathrm{~dB}$ with an $\mathrm{EAB}$ of $3.7 \mathrm{GHz}$ at $3 \mathrm{~mm}$ [17]. Li et al. fabricated MXene/Ni nanocomposites through the electrostatic self-assembly interaction, which achieved a $\mathrm{RL}_{\min }$ of $-50.5 \mathrm{~dB}$ with a narrow $\mathrm{EAB}$ at $2.5 \mathrm{~mm}$ [18].

Generally, the structure design of absorbing materials improved EM wave attenuation, such as porous, flower-like, core-shell, and hollow structures [19, 20]. Cui et al. obtained porous flower-like MXene/Ni composite microspheres, which show a $\mathrm{RL}_{\text {min }}$ value of $-52.7 \mathrm{~dB}$ [21]. Sheng et al. fabricated hierarchical core-shell structure $\mathrm{rGO} / \mathrm{Ni}$ nanofibers with a $\mathrm{RL}_{\min }$ of $-50.52 \mathrm{~dB}$ [22]. Zhu et al. synthesized highly cross-linked 3D structure $\mathrm{Fe} / \mathrm{Fe}_{2} \mathrm{O}_{3} @$ porous carbon composites with a $\mathrm{RL}_{\text {min }}$ of $-54.7 \mathrm{~dB}$ [23]. Gu et al. synthesized hybrid MF, which shows $\mathrm{RL}_{\min }$ of $-59.8 \mathrm{~dB}$ with the thickness of $2.3 \mathrm{~mm}$, and $\mathrm{EAB}$ of the maximum $5.64 \mathrm{GHz}$ at the thickness of $2.1 \mathrm{~mm}$ [24]. Su et al. synthesized $\mathrm{SiC}_{\mathrm{nw}} @$ $\mathrm{SiC}$ foam, and achieved a $\mathrm{RL}_{\min }$ of $-52.49 \mathrm{~dB}$ and $\mathrm{EAB}$ of $5.6 \mathrm{GHz}$ at a thickness of $2.82 \mathrm{~mm}$ [25]. These results indicate that the internal structure of the absorbing material could affect its absorption performance. Among them, the 3D porous structure of MF could endow the absorbing material with the advantages of low density, rich interface polarization, multiple reflections and scattering, thereby attenuating more EM waves [26]. Therefore, combining the highly conductive MXene and magnetic flower-like metal with the 3D porous structure could not only improve the excess electrical conductivity and complex permittivity, and enhance impedance matching, but also provide abundant interfaces and dipole polarization, increase the EM wave multiple reflections and scattering, and is considered a promising strategy to achieve excellent EM absorption property.

To meet the application requirements of the ever-increasing complex environment, advanced absorbing materials must possess multifunctional characteristics in addition to high-efficiency absorbing performance, such as flame retardancy, heat insulation, and infrared stealth, need to be considered [27, 28]. The long-term exposure of the absorbing material to the EM environment will convert the EM wave energy into heat energy, causing local temperature rise, which required the absorbing material to have the requirements of heat insulation and flame retardancy $[29,30]$. Furthermore, stealth materials that combine EM wave absorption and infrared shield have broader application prospects in both civilian and military applications [31]. Accordingly, due to the diversity of its actual application environment, it is imperative to develop microwave absorbing materials with thermal insulation, flame-retardant, and infrared stealth.

Herein, we successfully synthesized the two-dimensional hybrids containing flower-like Ni and few-layered MXene through electrostatic self-assembly. Then, the Ni flower/ MXene hybrids were assembled on the surface of MF (Ni/ MXene-MF) by dipping method, achieving high-efficiency microwave absorption and multifunctionality. Thanks to proper impedance matching, abundant polarization, the synergistic effect of dielectric loss and magnetic loss, as well as multiple scattering and reflection, Ni/MXene-MF exhibits strong microwave absorption $\left(\mathrm{RL}_{\min }\right.$ of $\left.-62.7 \mathrm{~dB}\right)$ corresponding to $\mathrm{EAB}$ of $6.24 \mathrm{GHz}$ at $2 \mathrm{~mm}$, and $6.88 \mathrm{GHz}$ at $1.8 \mathrm{~mm}$, covering the entire $\mathrm{Ku}$ band. In addition, good 
flame retardant, heat insulation, and infrared stealth functions make Ni/MXene-MF have broad application prospects in complex environments. This work provided a novel strategy for the development of multifunctional microwave absorbing materials that meet practical applications.

\section{Experiment Section}

\subsection{Materials}

$\mathrm{Ti}_{3} \mathrm{AlC}_{2}$ MAX powders were obtained by 11 Technology Co. MF was provided by Chengdu Yulong Chaoju New Material Co. Ltd. Lithium fluoride (LiF), Tris(hydroxymethyl)aminomethane, dopamine hydrochloride, ethylene glycol (EG), nickel chloride hexahydrate $\left(\mathrm{NiCl}_{2} \cdot 6 \mathrm{H}_{2} \mathrm{O}\right)$, sodium hydroxide $(\mathrm{NaOH})$, and ethanol were purchased from Shanghai Aladdin Co., Ltd. Hydrogen chloride $(\mathrm{HCl})$ was obtained from Sinopharm Chemical Reagent Co.

\subsection{Synthesis of $\mathrm{Ti}_{3} \mathrm{C}_{2} \mathrm{~T}_{\mathrm{x}}$ MXene}

First, $2 \mathrm{~g}$ of LiF was added into $40 \mathrm{~mL}$ of $9 \mathrm{M} \mathrm{HCL}$ and stirred for 30 min until the LiF powder was dissolved. Then, $2 \mathrm{~g}$ of $\mathrm{Ti}_{3} \mathrm{AlC}$ powder was slowly added to the mixed solution, the reaction temperature was controlled at $35^{\circ} \mathrm{C}$, and the stirring was continued for $24 \mathrm{~h}$. Then, the obtained suspension was washed several times with deionized (DI) water until its $\mathrm{pH}$ reached 6 , and then ethanol (the effect of intercalant) was added to ultrasonic centrifugation. Finally, the upper dispersion was collected after ultrasonic centrifugation with DI water and freeze-dried to obtain MXene sheets.

\subsection{Synthesis of Ni Flower}

Ni flower were prepared via a facile hydrothermal process. Firstly, $1.18 \mathrm{~g}$ of $\mathrm{NiCl}_{2} \cdot 6 \mathrm{H}_{2} \mathrm{O}$ and $3.6 \mathrm{~g}$ of $\mathrm{NaOH}$ were dissolved into $200 \mathrm{~mL}$ of EG. Then, it is stirred for $60 \mathrm{~min}$ until a uniform light green solution was formed. Finally, the precursor liquid was poured into the hydrothermal reactor and reacted at $200{ }^{\circ} \mathrm{C}$ for $10 \mathrm{~h}$. After washing the black precipitate with deionized ethanol and water several times, dry the collected Ni flower in a vacuum drying oven for $12 \mathrm{~h}$.

\subsection{Preparation of Ni Flower/MXene Hybrids}

Ni flower/MXene hybrid is prepared by electrostatic self-assembly process. First, $30 \mathrm{mg}$ of Ni flower was added to the CTAB solution $\left(2 \mathrm{mg} \mathrm{mL}^{-1}\right)$, and ultrasound for $1 \mathrm{~h}$ to make CTAB adsorb to the surface of Ni flower. Then, the Ni flower modified by centrifugation was washed by adding deionized water to remove excess CTAB. Finally, add the positively charged Ni flower to a few-layered MXene solutions (Ni flower: MXene $=1$ : 2 ). The prepared mixture used a shaker to shake for $24 \mathrm{~h}$ to ensure that the positively charged Ni flower and the negatively charged MXene are fully assembled electrostatically. Finally, the $\mathrm{Ni}$ flower/MXene hybrid was collected by centrifugation process and dried in a freeze dryer.

\subsection{Preparation of Ni/MXene-MF}

First, the MF was ultrasonically cleaned several times with deionized water and absolute ethanol, and it was dried and ready for use. First, the surface of MF was modified by PDA (the modification method in Supporting information) to increase the surface adhesion of MF. The modified MF was immersed in $2 \mathrm{mg} \mathrm{mL}^{-1}$ Ni flower, MXene, and Ni flower/MXene suspensions for $1 \mathrm{~min}$, respectively, then dried at $80^{\circ} \mathrm{C}$ to remove excess water, and the above dip-coating process was repeated three times.

\subsection{Characterization}

The crystal structure was identified by XRD (Bruker D8 Advance XRD) using Cu K radiation (1.5604 $\AA$ ). The surface chemical compositions were analyzed by X-ray photoelectron spectroscopy (XPS, Thermo Escalab 250Xi X). The microscopic morphology and structure were observed using SEM (SEM, Zeiss Sigma 300) and transmission electron microscopy (TEM, FEI Tecnai G2 F20). The magnetic properties of samples were measured by a vibrating sample magnetometer (VSM, 7404, LakeShore, USA) at room temperature. The chemical structure was characterized by Fourier transform infrared spectroscopy (FTIR, Nicolet 6700). The infrared radiation intensity of the composite foam was measured by a thermal imaging camera (E60, FLIR). To measure the EM absorption performance, the obtained 20 $\mathrm{wt} \%$ composites were mixed with $80 \mathrm{wt} \%$ paraffin were cut into concentric rings with an inner diameter of $3.04 \mathrm{~mm}$ and outer diameter of $7.00 \mathrm{~mm}$. Then, EM parameters were 
(a)

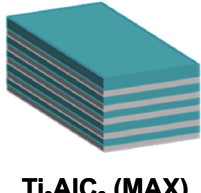

$\mathrm{Ti}_{3} \mathrm{AlC}_{2}$ (MAX)
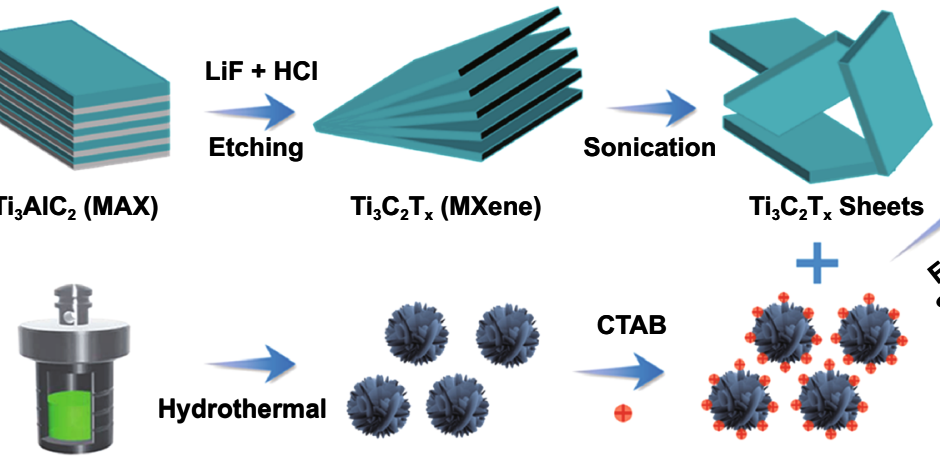

Ni flowers

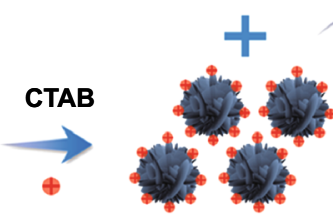

Ni flowers with positive charge

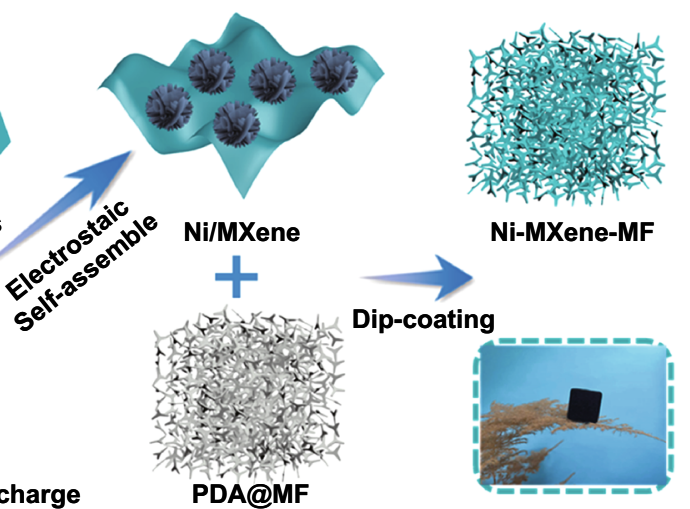

PDA@MF
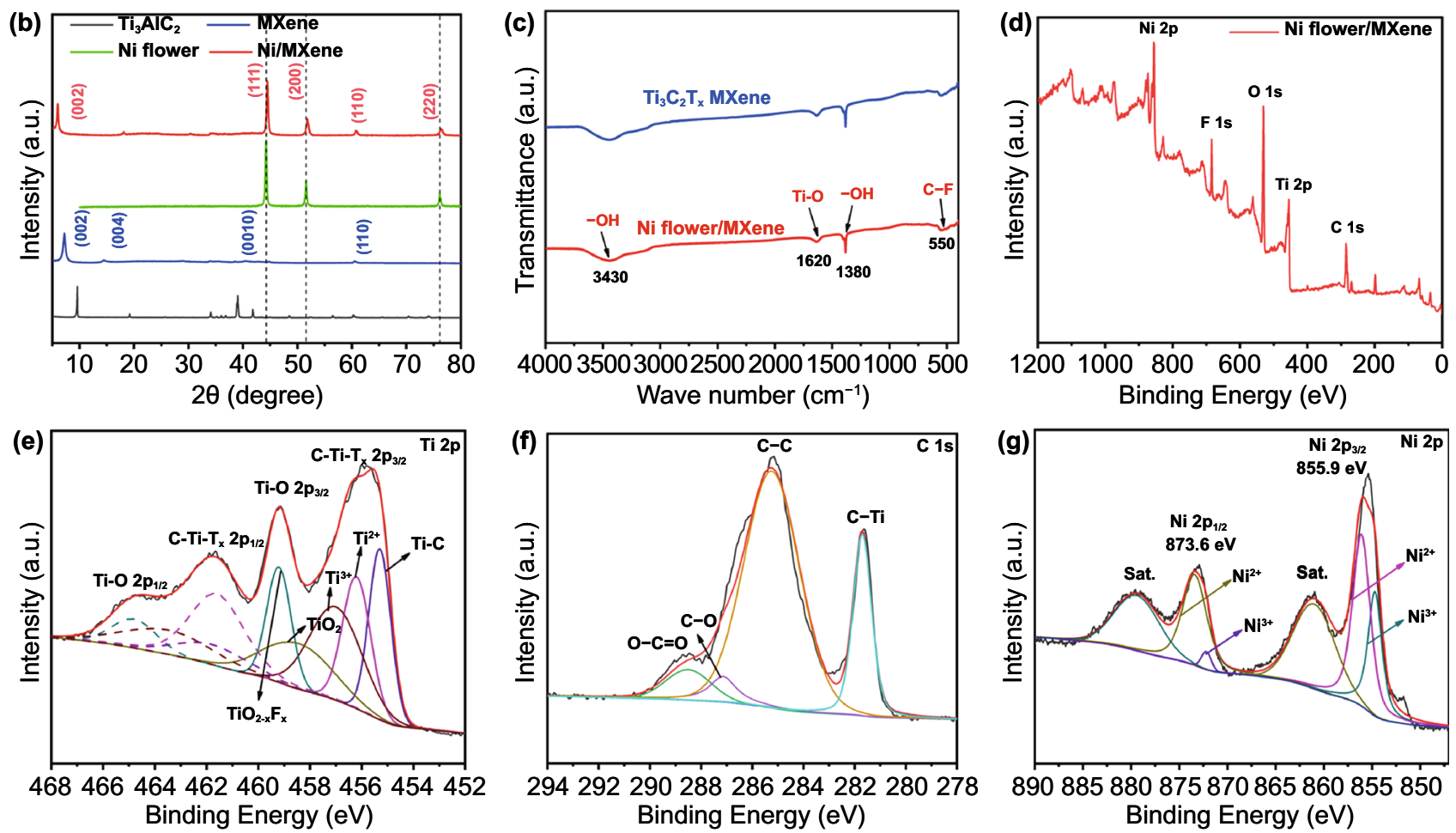

Fig. 1 a Schematic illustration of preparation process for Ni/MXene-MF. b XRD patterns of $\mathrm{Ti}_{3} \mathrm{AlC}_{2}, \mathrm{Ti}_{3} \mathrm{C}_{2} \mathrm{~T}_{\mathrm{x}}, \mathrm{Ni}$ flower, and Ni/MXene hybrid. C FTIR spectra of $\mathrm{Ti}_{3} \mathrm{C}_{2} \mathrm{~T}_{\mathrm{x}}$ and Ni/MXene hybrid. The XPS of spectra of $\mathbf{d ~ N i / M X e n e , ~} \mathbf{e} \mathrm{Ti} 2 \mathrm{p}, \mathbf{f} \mathrm{C} 1 \mathrm{~s}$, and $\mathbf{g}$ Ni 2p

measured with the coaxial line method by a vector network analyzer (VNA; Agilent 5324A).

\section{Results and Discussion}

\subsection{Chemical Structure and Morphology}

The detailed fabrication process of Ni/MXene-MF is shown in Fig. 1a. First, $\mathrm{Ti}_{3} \mathrm{AlC}_{2}$ was etched by LiF-HCL to remove $\mathrm{Al}$ atomic layers, and then the accordion-like MXene was ultrasonically processed to obtain a few-layered MXene. Due to its abundant functional groups, the obtained MXene flakes exhibit negative zeta potential [32]. Secondly, a positive charged Ni flower with good dispersibility was synthesized through hydrothermal reaction and CTAB modification. Then, the positively charged $\mathrm{Ni}$ was added to the negatively charged MXene. During the shaking process, the Ni was anchored on the surface of MXene through electrostatic interaction. Finally, the PDA-modified MF was immersed into Ni/MXene solution. During dip-coating processes, the $\mathrm{Ni} / \mathrm{MXene}$ hybrids were assembled on the surface of MF 
through the capillary force of MF and the adhesive power of PDA. The Ni/MXene-MF was obtained after drying to remove excess water.

The XRD patterns of $\mathrm{Ti}_{3} \mathrm{AlC}_{2}$, MXene, Ni flower, and Ni/MXene hybrid are shown in Fig. 1b. The disappearance of the diffraction peak of $\mathrm{Ti}_{3} \mathrm{C}_{2} \mathrm{~T}_{\mathrm{x}}$ MXene at $39^{\circ}$ indicated the $\mathrm{Al}$ atomic layers were removed [33]. Meanwhile, the (002) diffraction perk shifted to a lower angle from $9.7^{\circ}$ to $7.6^{\circ}$, which confirmed the widen $d$-spacing of $\mathrm{Ti}_{3} \mathrm{C}_{2} \mathrm{~T}_{\mathrm{x}}$ MXene sheets. The observed peaks at $44.7^{\circ}, 51.8^{\circ}$, and $76.8^{\circ}$ in the XRD pattern of Ni were perfectly indexed to (111), (200), and (200) crystal faces of cubic crystalloid structure (PDF \#03-1051) [34]. For the Ni/MXene hybrid, the typical diffraction peaks of $\mathrm{Ni}$ and MXene could be obviously observed, indicating the successful fabrication of the $\mathrm{Ni} /$ MXene hybrid. Besides, the (002) surface peak belonging to MXene in the XRD pattern of the Ni/MXene hybrid was shifted to a lower angle from $7.6^{\circ}$ to $6.1^{\circ}$, indicating that the MXene layer d-spacing increased, which could be attributed to the insertion of $\mathrm{Ni}$ particles or the successful peeling of the few-layered MXene during the electrostatic assembly process.

The XPS was used to prove element composition and valence states information. It revealed that MXene contains Ti, C, O, and F elements (Fig. S1), while Ni/MXene mainly contains $\mathrm{Ni}, \mathrm{Ti}, \mathrm{C}, \mathrm{O}$, and $\mathrm{F}$ elements in the spectrum, verifying the combination between Ni flower and MXene sheets (Fig. 1d). The Ti 2p related four peaks of Ni/MXene at 465.1, 461.6, 459.8, and $455.7 \mathrm{eV}$ correspond to Ti-O 2 $\mathrm{p}_{1 / 2}$ bonds, C-Ti-T ${ }_{x} 2 p_{1 / 2}$, Ti-O $2 p_{3 / 2}$, and C-Ti-T ${ }_{x} 2 p_{3 / 2}$, respectively (Fig. 1e) [35]. In addition, four obvious peaks found in the $\mathrm{C} 1 \mathrm{~s}$ spectrum at $289.1,286.5,285.1$, and $281.8 \mathrm{eV}$ correspond to $\mathrm{O}-\mathrm{C}=\mathrm{O}, \mathrm{C}-\mathrm{O}, \mathrm{C}=\mathrm{C}$, and $\mathrm{C}-\mathrm{Ti}-\mathrm{T}_{\mathrm{x}}$ bonds, respectively (Fig. 1f) [36]. The Ni 2p spectrum included two main peaks at 873.6 and $855.9 \mathrm{eV}$, which correspond to $\mathrm{Ni} 2 \mathrm{p}_{1 / 2}$ and $\mathrm{Ni} 2 \mathrm{p}_{3 / 2}$, indicating that Ni flower was successfully introduced [22]. In addition, FTIR spectroscopy also confirmed the existence of functional groups similar to MXene in the Ni/MXene hybrid, the peaks at 550, 1620, and $3430 \mathrm{~cm}^{-1}$ correspond to $\mathrm{C}-\mathrm{F}, \mathrm{Ti}-\mathrm{O}$, and $-\mathrm{OH}$ bonds (Fig. 1c) [37, 38]. These abundant functional groups could cause dipole and defect polarization, thereby enhancing the EM attenuation ability.

The morphologies of the few-layered MXene, Ni flower, and Ni/MXene hybrid were observed through the SEM and TEM. The few-layered MXene was observed a wrinkled film-like structure (Fig. 2a). Ni flower possessed a distinct petal-like structure, and its average size is about $1 \mu \mathrm{m}$ (Fig. 2b). As for the Ni/MXene hybrid, the Ni flowers were evenly anchored on the surface of MXene or wrapped by MXene (Fig. 2c-d). The HR-TEM image of the Ni/MXene hybrid further revealed the interface and crystal structure, the interface lattice between Ni flower and MXene indicated that good coalescence occurred at the atomic level. In addition, the d-spacing $(0.2021)$ of periodic lattice fringes corresponded to the crystal Ni with face-centered cubic (111) (Fig. 2e), which is consistent with the XRD results. The element mapping image (Fig. 2f) of the Ni/MXene hybrid confirmed the uniform distribution of $\mathrm{Ni}, \mathrm{Ti}$, and $\mathrm{C}$. The synthesized MXene, Ni flower, and Ni/MXene hybrid were assembled on the surface of MF through the dip-coating adsorption process. Compared with the smooth surface of the MF, the MXene-MF skeleton and the surrounding area have a few-layered MXene successfully assembled (Fig. 2g), and the Ni flower-MF skeleton has a large number of uniform and dense particles (Fig. 2h). For the Ni/MXene-MF, the $\mathrm{Ni} / \mathrm{MXene}$ hybrid wrapped on the MF skeleton tightly and homogeneously (Fig. 2i). Among them, through the electrostatic self-assembly process, the magnetic Ni flower modified on the surface of MXene could produce a large number of interfaces and form a "micro-capacitor" structure, which was conducive to improving impedance matching and generating interface polarization. In addition, the three-dimensional conductive network formed by the $\mathrm{Ni} /$ MXene hybrid along the MF framework help to increase the electron transmission rate and enhance the conductance loss. The porous structure of MF is conducted to EM waves multiple reflect, scatter, and absorb.

\subsection{Microwave Absorption}

The EM parameters are the key to microwave absorption performance, the complex permittivity $\left(\varepsilon_{r}=\varepsilon^{\prime}-j \varepsilon^{\prime \prime}\right)$, and the complex permeability $\left(\mu_{r}=\mu^{\prime}-j \mu^{\prime \prime}\right)$. The real parts $\varepsilon^{\prime}$ and $\mu^{\prime}$ represent the storage capacity of EM wave energy, and the imaginary parts $\varepsilon^{\prime \prime}$ and $\mu^{\prime \prime}$ represent the dissipation ability $[39,40]$. The EM parameters of MXene-MF, Ni/MXene-MF, and Ni-MF frequency-dependent are under the same filler loading ratio, as shown in Fig. 3. It is found that the permittivity decreased as the frequency increased, which can be attributed to the frequency dispersion effect. Among them, 

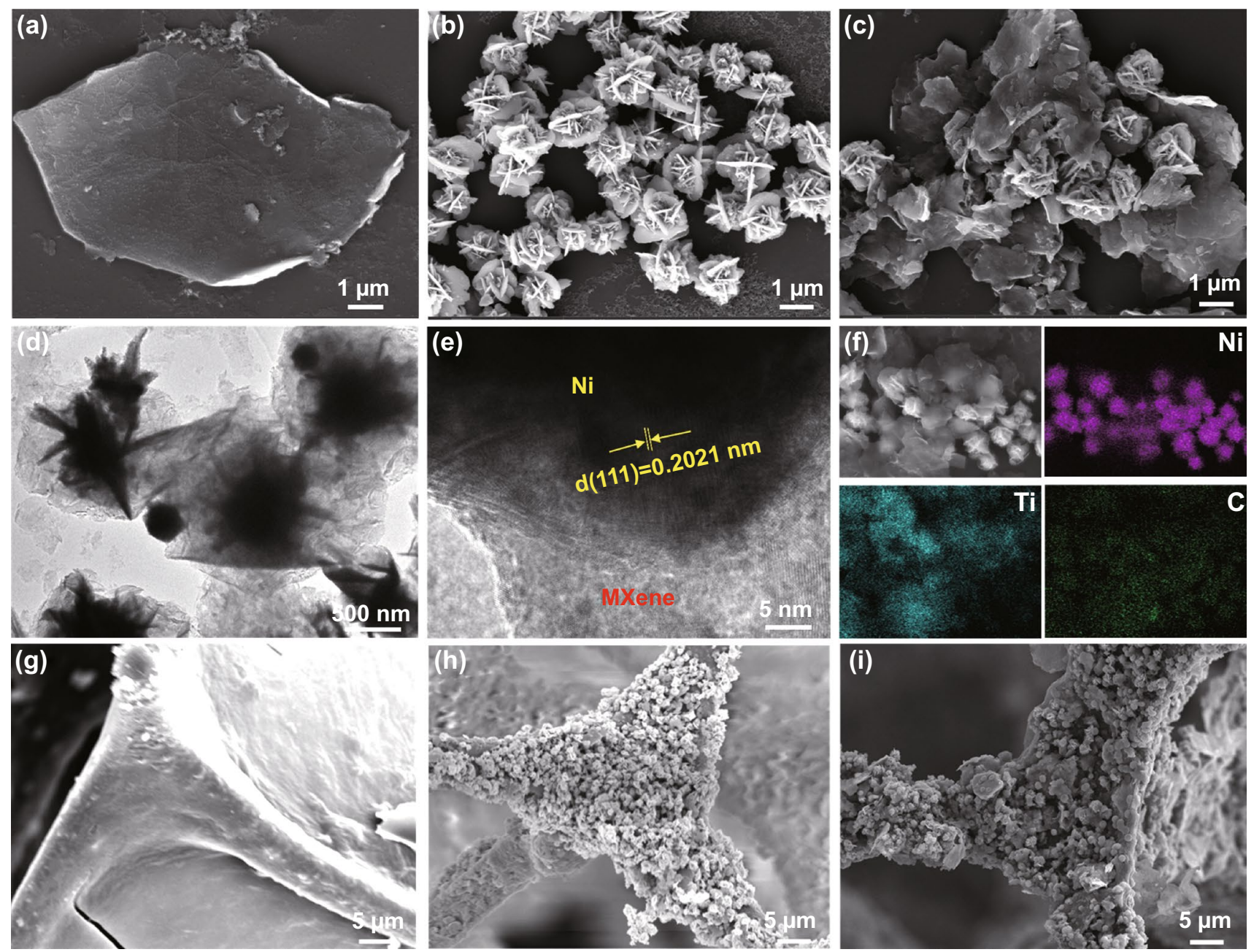

Fig. 2 SEM images of a $\mathrm{Ti}_{3} \mathrm{C}_{2} \mathrm{~T}_{\mathrm{x}}$, b Ni flower, $\mathbf{c} \mathrm{Ni} / \mathrm{MX}$ ene hybrids, $\mathbf{g}$ MXene-MF, $\mathbf{h}$ Ni flower-MF, and i Ni/MXene-MF. d TEM, e HR-TEM images and $\mathbf{f}$ the corresponding elemental mapping images of $\mathrm{Ni} / \mathrm{MX}$ ene hybrid

MXene-MF exhibited a high complex dielectric constant due to the ultra-high conductivity of MXene, the values of $\varepsilon^{\prime}$ and $\varepsilon^{\prime \prime}$ declined from 13.5 to 5.7 and 7.9 to 3.6 in the frequency range of $2-18 \mathrm{GHz}$, respectively. As for Ni flower-MF, the values of $\varepsilon^{\prime}$ and $\varepsilon^{\prime \prime}$ were low, declined from 5.7 to 3.4 and 2.6 to 1.1 , respectively, indicating that Ni flower/MF possessed poor storage and dissipation capabilities of EM energy. After the electrostatic self-assembly of Ni flower and MXene, the $\varepsilon^{\prime}$ and $\varepsilon^{\prime \prime}$ values of the Ni/MXene-MF obtained were 8.4 to 4.3 and 5.9 to 2.1 , respectively. It was worth noting that the $\varepsilon^{\prime \prime}$ value of Ni/MXene-MF was smaller than that of MXene-MF. Generally, according to the classic free electron theory $\left(\varepsilon^{\prime \prime}=\frac{\varepsilon_{s}-\varepsilon_{\infty}}{1+2 \pi f^{2} \tau^{2}} 2 \pi f \tau+\frac{\sigma}{2 \pi f \varepsilon_{0}}\right)$, the value of $\varepsilon^{\prime \prime}$ increased as the value of conductivity increased [41]. Therefore, compared with MXene-MF, due to the introduction of non-conductive Ni flower and the reduction in MXene content, the conductivity $(\sigma)$ of Ni/MXene-MF decreased, resulting in a decrease in $\varepsilon^{\prime \prime}$ value. The appropriate $\varepsilon^{\prime \prime}, \varepsilon^{\prime}$ and $\sigma$ of Ni/MXene-MF were conducive to better impedance matching and could enhance the EM absorption capacity.

Generally, the complex permeability was described as: $\mu^{\prime}=1+\frac{M}{H} \cos \theta$ and $\mu^{\prime \prime}=1+\frac{M}{H} \sin \theta$. Here, $\mathrm{M}, \mathrm{H}$, and $\theta$ were magnetization, external magnetic field, and phase lag angle of $\mathrm{M}$ behind $\mathrm{H}$, respectively [42]. Due to the nonmagnetic nature of MXene, the $\mu^{\prime}$ value of MXene-MF was around 1.05 , and the $\mu^{\prime \prime}$ value was around 0 . However, the values of $\mu^{\prime}$ and $\mu^{\prime \prime}$ of Ni/MXene-MF and Ni flower-MF were slightly increased due to the presence of magnetic $\mathrm{Ni}$ flower. Among them, the saturated magnetization values of $\mathrm{Ni} / \mathrm{MXene-MF}$ and Ni flower-MF were $30.8 \mathrm{emu} \mathrm{g}^{-1}$ and 

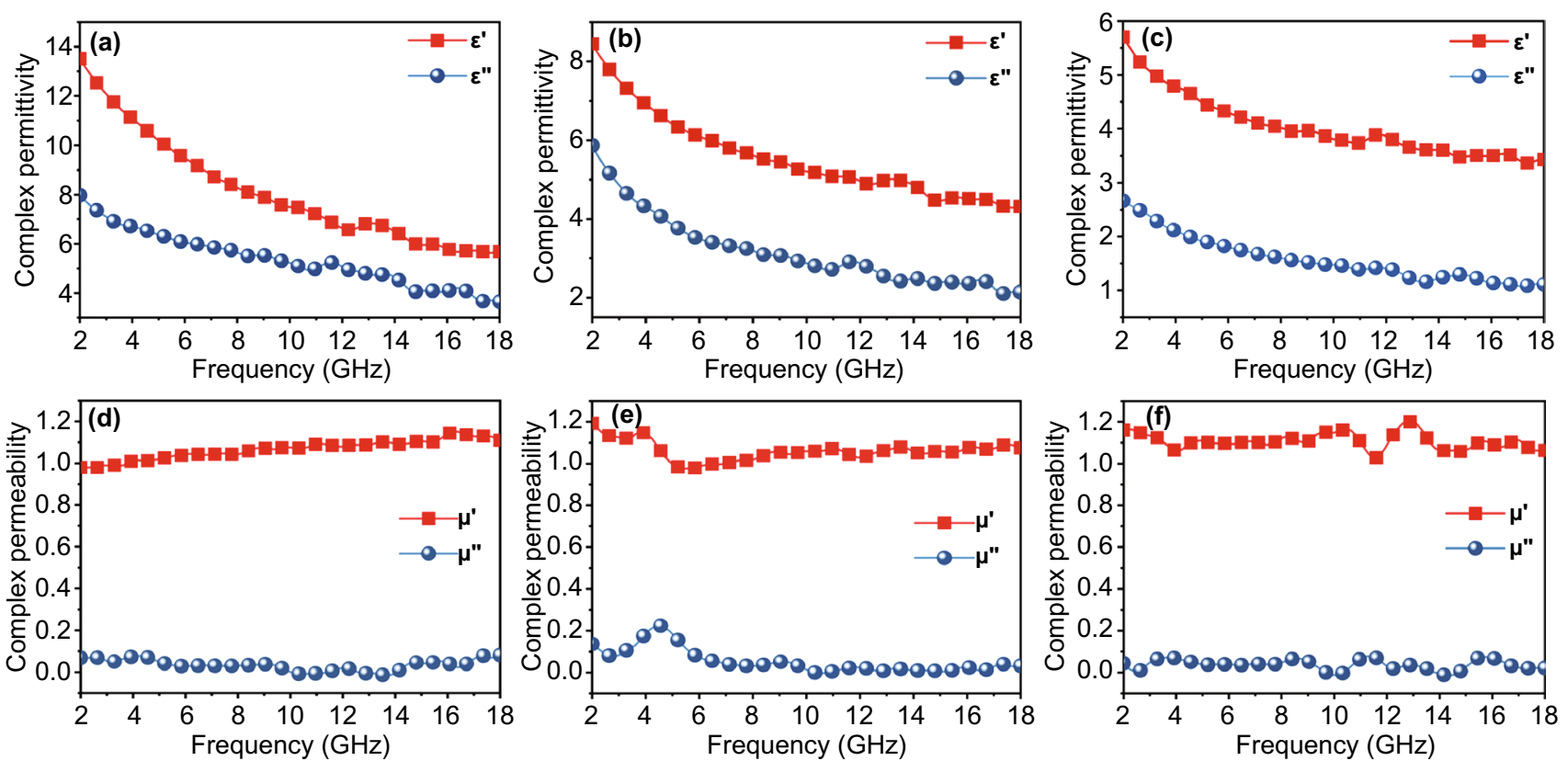

Fig. 3 Electromagnetic parameters $\left(\varepsilon^{\prime}, \varepsilon^{\prime \prime}, \mu^{\prime}\right.$ and $\left.\mu^{\prime \prime}\right)$ for a, d MXene-MF, b, e Ni/MXene-MF and c, f Ni flower-MF

$18.5 \mathrm{emu} / \mathrm{g}$, respectively (Fig. S3a). Generally, the eddy current effect could be evaluated according to the $\mathrm{C}_{0}$ value [43]. The calculation formula of the $\mathrm{C}_{0}$ value was as follows:

$C_{0}=\mu^{\prime \prime}\left(\mu^{\prime}\right)^{-2} f^{-1}$

If the value of $\mathrm{C}_{0}$ remained constant over the entire frequency range, eddy current loss was the only source of magnetic loss [44]. As shown in Fig. S3b, the $\mathrm{C}_{0}$ value of $\mathrm{Ni}$ / MXene-MF and Ni flower-MF fluctuates with frequency, indicating that the magnetic loss mainly includes natural resonance and exchange resonance.

Since N Ni/MXene-MF possessed numerous heterogeneous interfaces and abundant functional groups, the dielectric loss mechanism was explored. The dielectric loss usually included polarization loss and conduction loss. The polarization loss could be divided into interface polarization and dipolar polarization. According to the Debye relaxation model, the type of dielectric loss could be described by the relationship between $\varepsilon^{\prime}$ and $\varepsilon^{\prime \prime}$. The calculation equation is as follows [45]:

$\left(\varepsilon^{\prime}-\frac{\varepsilon_{s}+\varepsilon_{\infty}}{2}\right)^{2}+\left(\varepsilon^{\prime \prime}\right)^{2}=\left(\frac{\varepsilon_{s}-\varepsilon_{\infty}}{2}\right)^{2}$
Among them, $\varepsilon_{s}$ and $\varepsilon_{\infty}$, respectively, represent static dielectric constant and relative dielectric constant. Usually, a Cole-Cole semicircle in the above formula corresponds to a Debye relaxation [46]. As shown in Fig. S4a-c, there are several small semicircles and one long tail in all three types of foams, indicating the coexistence of polarization loss and conduction loss. Specifically, multiple heterogeneous interfaces between Ni flower, MXene, MF, and air promote interfacial polarization, a large number of functional groups and lattice defects in MXene cause dipole polarization, and the MF skeleton provides a transport path for electron motion, causing conductive loss.

EM absorption performance could be intuitively evaluated by reflection loss (RL), and the RL was calculated according to the transmission line theory as:

$R L=20\left|\frac{Z_{\text {in }}-Z_{0}}{Z_{\text {in }}+Z_{0}}\right|$

$Z_{\text {in }}=Z_{0} \sqrt{\frac{r}{r}} \tanh \left(j \frac{2 f d}{c} \sqrt{r r}\right)$

where $Z_{\text {in }}$ is the normalized input impedance of the absorber, $\mathrm{Z}_{0}$ is the impedance of free space, $f$ is the frequency of the EM wave, $d$ is the thickness of the absorber, and $c$ is the velocity of light in free space $[9,47]$. Specifically, the RL 
value should be lower than $-10 \mathrm{~dB}$, and more than $90 \%$ of the incident EM waves will be attenuated [48]. Simultaneously, to visually reveal the relationship between RL value, frequency, and thickness, the $3 \mathrm{D}$ reflection loss diagrams of three different samples are shown in Fig. 4a-c. It could be seen intuitively that the RL value of MXene-MF, Ni/MXeneMF, and Ni flower-MF could be adjusted by changing the thickness (Fig. S5). However, a low RL value of MXene-MF and Ni flower-MF in the thickness range of 1-4 mm, and the adjustable thickness was limited, indicating that they were not suitable for practical applications of microwave absorption. In contrast, Ni/MXene-MF exhibited outstanding microwave absorption performance. In particular, a strong RLmin of $62.7 \mathrm{~dB}$ with the EAB of $6.24 \mathrm{GHz}$ at the ultrathin thickness of $2 \mathrm{~mm}$ was obtained. In addition, the EAB of the Ni/MXene-MF could reach $6.88 \mathrm{GHz}$ with a thickness of $1.8 \mathrm{~mm}$, which was from 11.12 to $18 \mathrm{GHz}$, as shown in Fig. 4d. Meanwhile, the relation between the absorber thickness $\left(t_{\mathrm{m}}\right)$ and the peak frequency $\left(f_{\mathrm{m}}\right)$ could be described by the quarter-wavelength $(1 / 4 \lambda)$ model as Eq. 5:
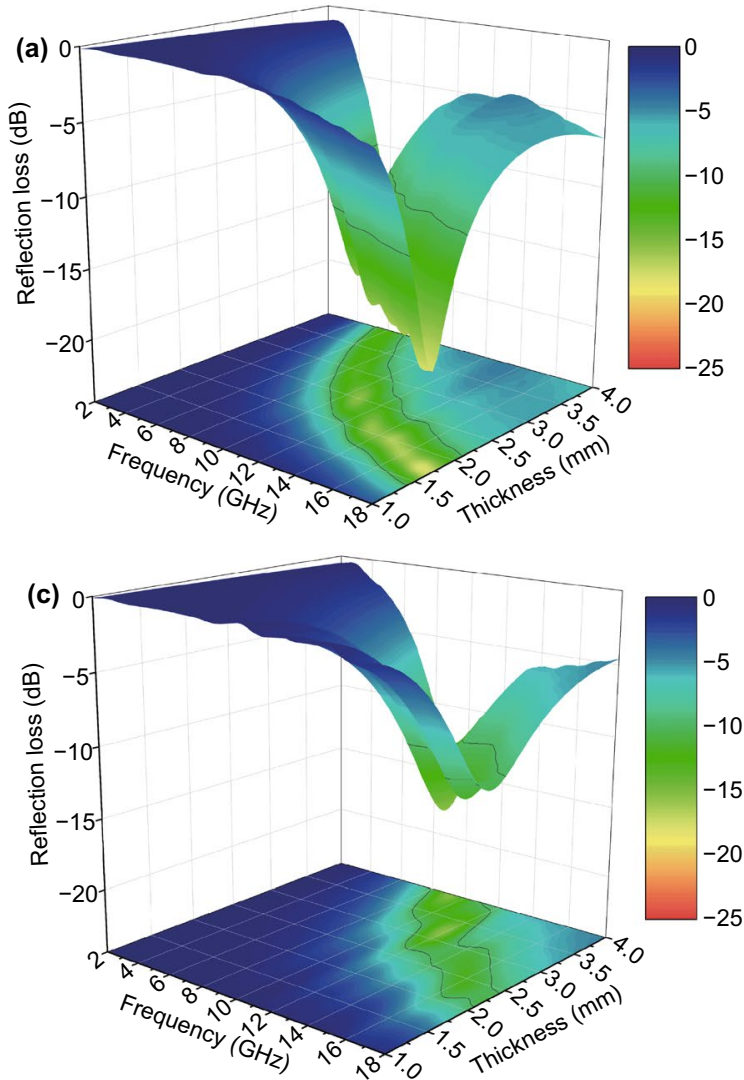

$t_{m}=\frac{n \lambda}{4}=\frac{n c}{4 f_{m} \sqrt{\left|\varepsilon_{r}\right|\left|\mu_{r}\right|}}(n=1,3,5, \ldots)$

The Ni/MXene-MF simulated curves of the RL peak frequency and the matching thickness well fits the quarterwavelength matching conditions (Fig. S6). The results show that Ni/MXene-MF possessed excellent EM wave absorption ability, and could be expected to exhibit great practical applications in the ongoing $5 \mathrm{G}$ communication technology.

Generally, impedance matching and attenuation were the basic factors affecting EM wave absorption performance [49]. Three processes occurred when EM waves were incident on the surface of the material: reflection, absorption, and transmission [50]. The adjustment of impedance matching could achieve reducing reflection and increasing absorption of incident EM wave. Here, the impedance matching was evaluated by introducing the $\left|Z_{\text {in }} / Z_{0}\right|$ value, which can be calculated based on Eq. 5:
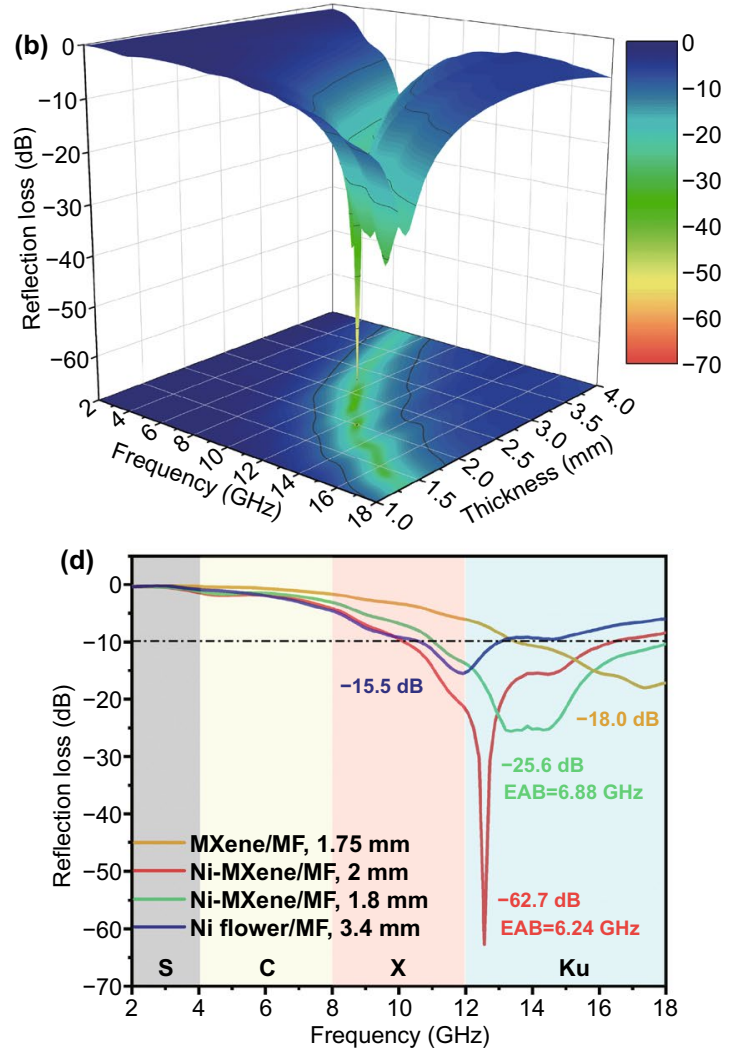

Fig. 4 Three-dimensional representations of RL values for a MXene-MF, b Ni/MXene-MF, and c Ni flower-MF. d Frequency-dependent RL values of MXene-MF-1.75 mm, Ni/MXene-MF-2 mm, Ni/MXene-MF-1.8 mm, and Ni flower-MF-3.4 mm 
$\frac{Z_{\text {in }}}{Z_{0}}=\sqrt{\frac{r}{r}} \tanh \left(j \frac{2 f d}{c} \sqrt{r r}\right)$

The value of $\left|Z_{\text {in }} / Z_{0}\right|$ should be equal or close to 1 (0.8-1.2), a large amount of EM wave could enter the absorber and be attenuated [51]. The 2D contour maps of the $\left|Z_{\text {in }}\right| Z_{0} \mid$ value of MXene-MF, Ni/MXene-MF, and Ni flower$\mathrm{MF}$ are shown in Fig. $5 \mathrm{a}-\mathrm{c}$, and the values close to 1 are marked with black lines. Among them, the $\left|Z_{\text {in }}\right| Z_{0} \mid$ value of MXene-MF was much higher than 1 , and the $\left|Z_{\text {in }} / Z_{0}\right|$ value of Ni flower-MF was much lower than 1, which lead to impedance mismatch, the large reflection of incident EM waves, and poor microwave absorption performance. By contrast, the black line of Ni/MXene-MF covered a broader frequency and wider thickness, and the $\left|Z_{\text {in }} / Z_{0}\right|$ value was close to 1 , achieving good impedance matching. The impedance matching was related to EM parameters, which were determined by the composition and structure of the absorber. Here, the impedance matching of Ni/MXene-MF was related to its
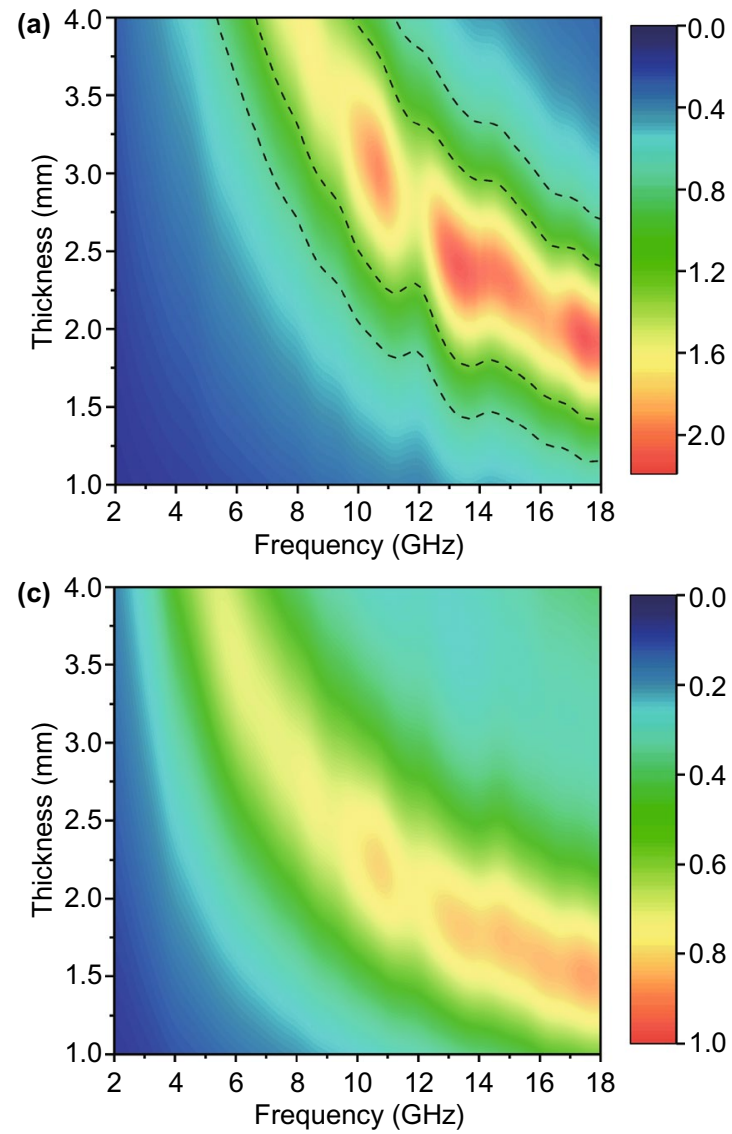

porous structure, the electrical conductivity from MXene, and the magnetic permeability of the Ni flower. In addition, the absorbed incident EM wave will be dissipated or converted into other energies. Here, the microwave attenuation capability of the sample was evaluated by introducing the attenuation constant $(\alpha)$, and the calculation formula is as follows [52]:

$\alpha=\frac{\sqrt{2}}{\mathrm{c}} \pi f \times \sqrt{\left(\mu^{\prime \prime} \varepsilon^{\prime \prime}-\mu^{\prime} \varepsilon^{\prime}\right)+\sqrt{\left(\mu^{\prime \prime} \varepsilon^{\prime \prime}-\mu^{\prime} \varepsilon^{\prime}\right)^{2}+\left(\mu^{\prime} \varepsilon^{\prime \prime}+\mu^{\prime \prime} \varepsilon^{\prime}\right)^{2}}}$

Figure 5d shows that the value of $\alpha$ gradually increased with increasing frequency. In particular, the $\alpha$ value changes in sequentially of Ni-MF (30.1-122.2) < Ni/MXene-MF (51.9-208.9) < MXene-MF (62.9-326.7). Based on Eq. 4, $\alpha$ was determined by the dielectric/magnetic loss capability. It could be seen that $\tan \delta_{\varepsilon}$ was significantly higher than $\tan \delta_{\mu}$ (Fig. S7), which indicated that the dielectric loss has
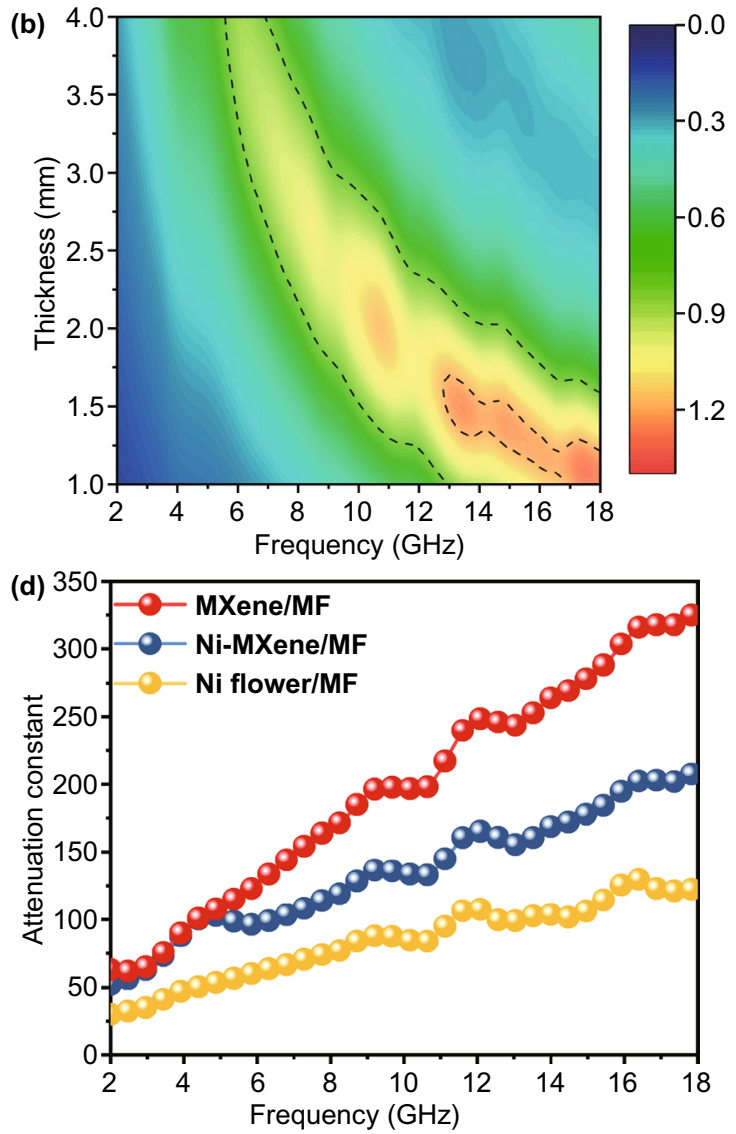

Fig. 5 Impedance matching for a MXene-MF, b Ni/MXene-MF, and $\mathbf{c}$ Ni flower-MF. $\mathbf{d}$ The frequency dependence of the attenuation constant $(\alpha)$ 
a greater effect on microwave attenuation than magnetic loss. However, the microwave absorption performance of the MXene/MF with the largest $\alpha$ value was not satisfactory, which shows that the attenuation ability cannot solely determine the microwave absorption of the absorbing material.

To further reveal the potential relationship among microwave absorption performance, impedance matching and attenuation capabilities, the frequency-dependent RL value, $\left|Z_{\text {in }} / Z_{0}\right|$, and $\alpha$ of Ni-MXene-MF are discussed and shown in Fig. 6a. The RL value did not achieve the minimum value as the $\alpha$ value increased to the maximum value of 208.9. As the $\left|\mathrm{Z}_{\text {in }}\right| \mathrm{Z}_{0} \mid$ value was close to 1 , the $\mathrm{RL}$ achieved the minimum value of $-62.7 \mathrm{~dB}$ at the frequency of $12.56 \mathrm{GHz}$. The above results show that Ni/MXene-MF achieved excellent microwave absorption properties through balancing impedance matching and attenuation efficiency. In addition, Fig. $6 \mathrm{~b}$ and Table S1 list the microwave absorption properties of related MF-based composites previously reported. Compared with other foam-based composite materials, Ni/MXene-MF possessed the advantages of a lightweight, thin matching $(2 \mathrm{~mm})$, wide EAB $(6.24 \mathrm{GHz})$, and strong absorption strength $(-62.7 \mathrm{~dB})$, and was regarded as an ideal choice for the EM wave absorbing materials.

\subsection{Microwave Absorption Mechanism}

The microwave absorption mechanism of Ni/MXene-MF is shown in Fig. 6c. Firstly, the 3D porous framework of $\mathrm{Ni} / \mathrm{MXene}-\mathrm{MF}$ could form an interconnected conductive
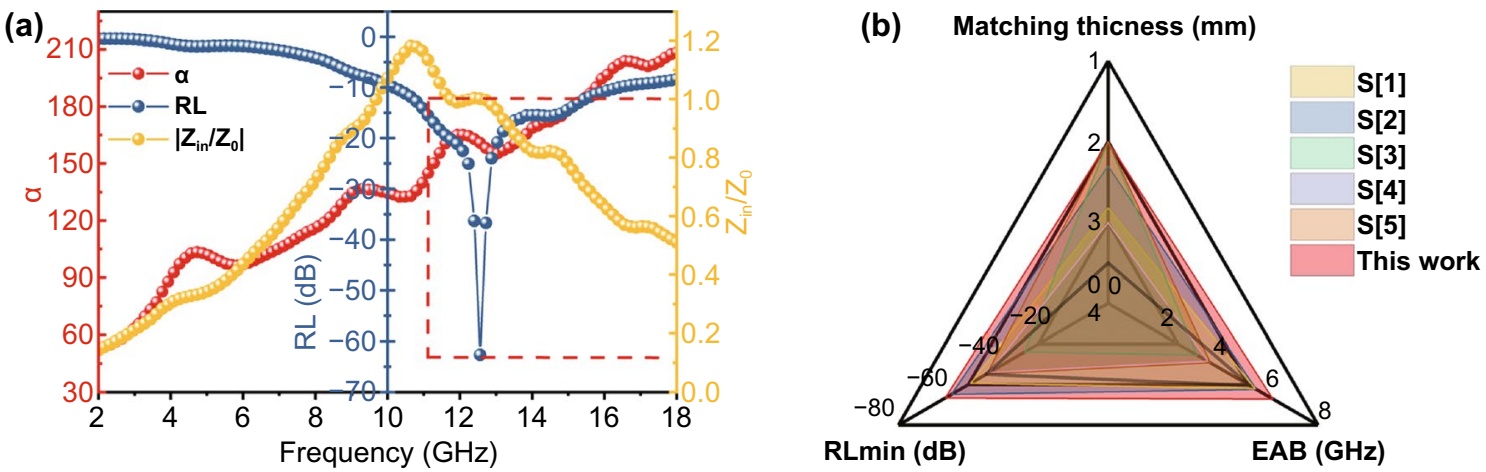

(c)

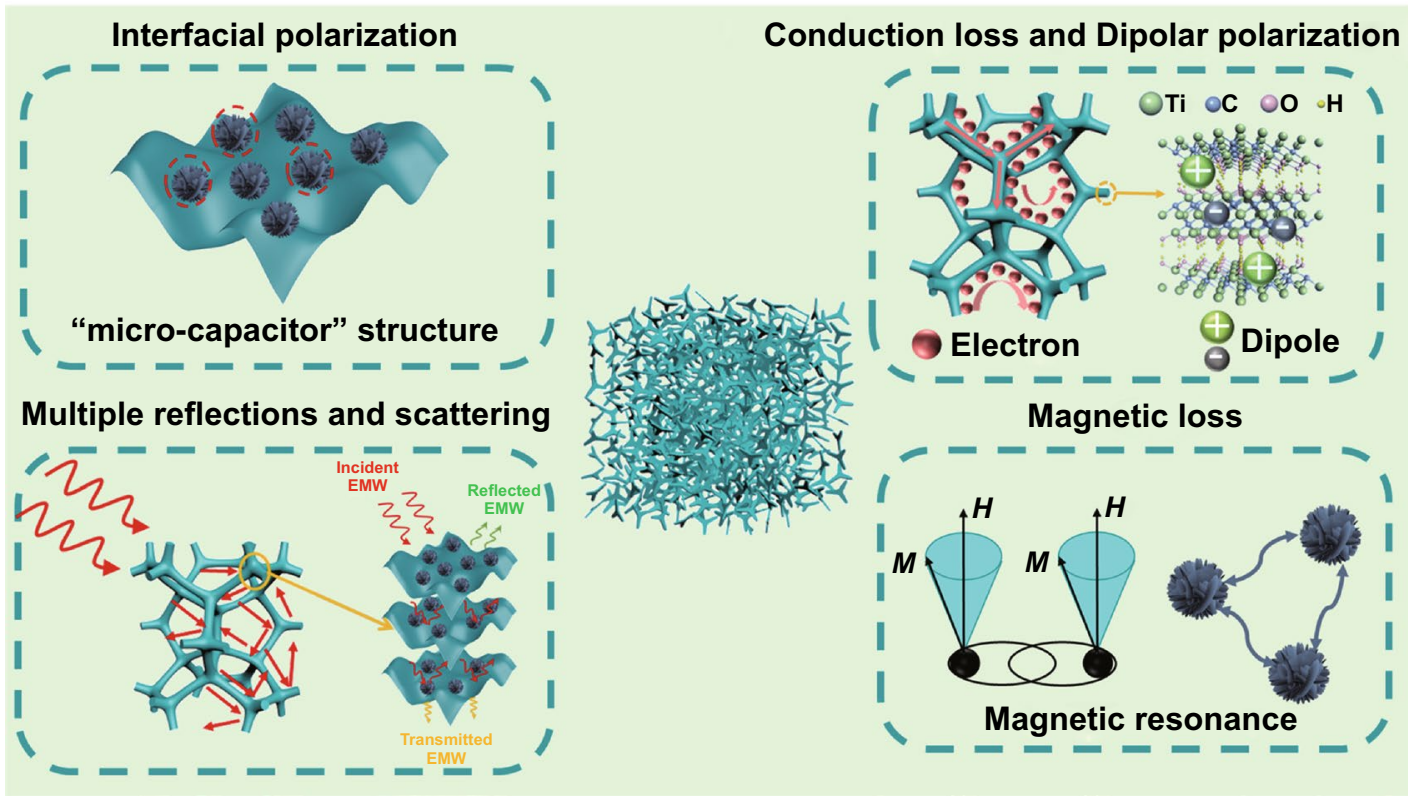

Fig. 6 a Frequency-dependent $\alpha, \mathrm{RL}$, and $\left|\mathrm{Z}_{\text {in }} / \mathrm{Z}_{0}\right|$ values of the Ni/MXene-MF-2 mm. b Comparison with the MA properties of the reported MF-based foam composites. $\mathbf{c}$ Schematic illustration of the microwave absorption mechanism of Ni/MXene-MF 
network, which could effectively help the migration of electrons, resulting in conduction losses and attenuating microwave energy [53-55]. Second, the Ni/MXene hybrid with a capacitor-like structure could provide a large number of interfaces, which could cause charge accumulation in the constantly changing EM field, generating interface polarization effects [56, 57]. In addition, abundant terminating functional groups and inherent defects of layered MXene will cause dipole polarization and defect polarization under highfrequency EM fields. Third, under the action of alternating EM fields, the Ni flower attenuated EM waves through natural resonance and exchange resonance [58-67]. Finally, the $3 \mathrm{D}$ porous network structure could increase the propagation path of microwaves in the foam, and the microwaves absorption efficiency could be improved after multiple reflection and scattering [68], while the microwaves also reflect and scatter between MXene and Ni flower.

\subsection{Multifunction}

To meet the increasing application requirements in complex environments, advanced absorbing materials need to possess multifunction, including heat insulation, infrared shielding performance, and flame retardancy [69-72]. Excellent heat insulation was an important function of absorbing materials, which could effectively protect electronic equipment or military weapon materials from overheating/overcooling temperature damage. The Ni/MXene-MF with a thickness of $1 \mathrm{~cm}$ was placed on a circular heating table platform at $80^{\circ} \mathrm{C}$. Meanwhile, the infrared thermal image of Ni/MXeneMF captured from 5 to $30 \mathrm{~min}$ is shown in Fig. 7a. The detection temperature on the upper surface of the sample was only $\sim 28.5, \sim 28.8, \sim 29.2$, and $29.3{ }^{\circ} \mathrm{C}$, respectively. It is shown that Ni/MXene-MF possesses excellent heat insulation and could effectively prevent heat transfer from the bottom to the upper surface. In addition, with the extension of heating time, the upper surface temperature of Ni/MXeneMF was almost unchanged, indicating that it possesses (a)

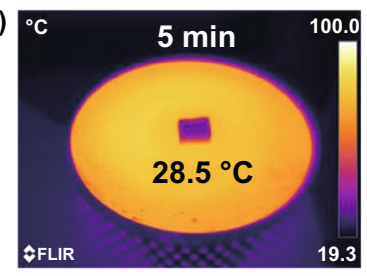

(b)

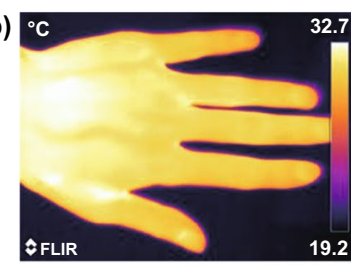

(c) ${ }^{\circ} \mathrm{C}$

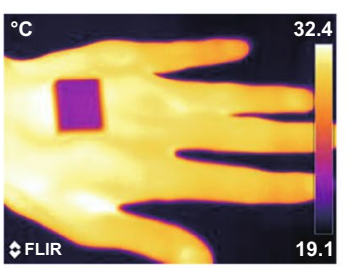

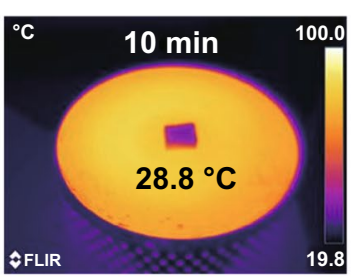
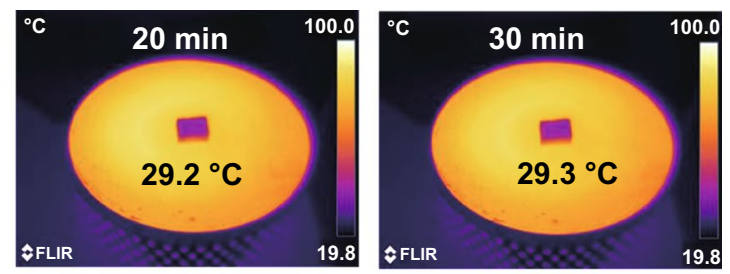

(d)

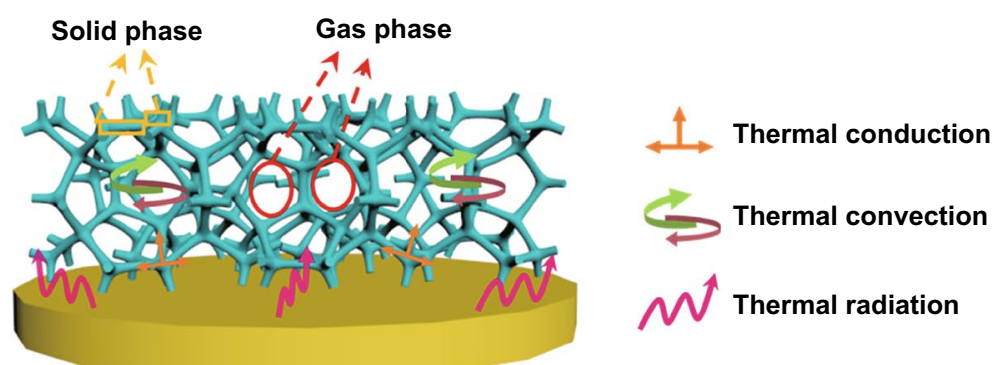

(e)
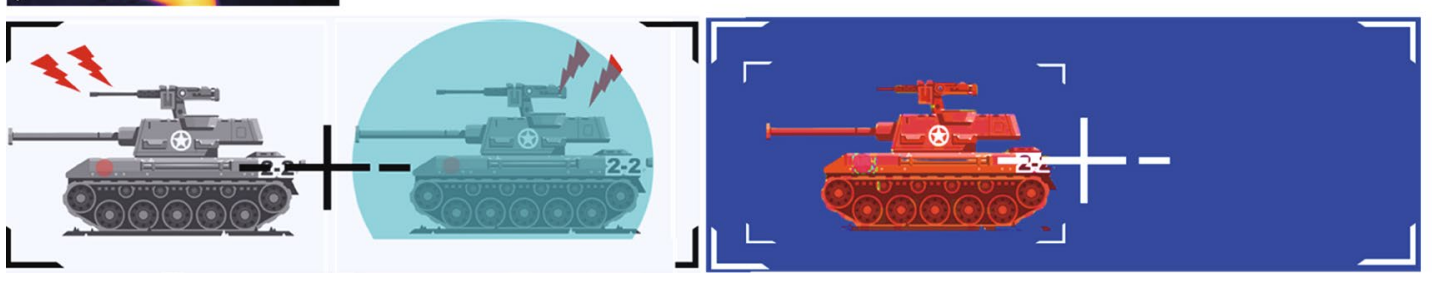

Fig. 7 Thermal infrared images of the Ni/MXene-MF placed a on the heating platform at different time under $80{ }^{\circ} \mathrm{C}$, and $\mathbf{b}-\mathbf{c}$ on the hand. The schematic diagram of $\mathbf{d}$ the heat transfer mechanism of Ni/MXene-MF, and $\mathbf{e}$ infrared stealth application 
excellent thermal insulation stability. More importantly, the superior heat insulation of MXene-MF made it possess an infrared stealth function, which could shield infrared radiation to hide military targets. Generally, objects with higher temperatures will show higher infrared radiation intensity under infrared thermal imaging. As shown in Fig. 7b, it could be seen that the color of the bare hand was different from the surrounding environment, making it visible under the infrared detection equipment. On the contrary, with a piece of Ni/MXene-MF was placed on the back of the hand, the color of the covered area was consistent with the color of the surrounding environment (Fig. 7c), making it disappear under the infrared detection equipment. Therefore, $\mathrm{Ni}$ / MXene-MF possesses strong infrared shielding properties. In addition, the flame retardancy of Ni/MXene-MF was verified through combustion experiments. As shown in Movie S1, MF possesses natural flame retardancy. When exposed to an open flame, the MF foam surface begins to burn and automatically extinguishes after leaving the flame, and without producing droplets. This is due to the MF rapidly forming dense coking on the burning surface and effectively preventing the combustion from developing to a deeper level. However, it cannot be used due to the shrinkage of the shape. On the contrary, Ni/MXene-MF maintained its original shape after burning (Movie S2). This is because the Ni/MXene hybrid was assembled on the surface of the MF to form a dense flame-retardant protective layer, and the high porosity could promote the rapid diffusion of heat during the combustion process.

The excellent heat insulation performance mechanism of Ni/MXene-MF is shown in Fig. 7d. The heat transfer methods of foam materials include heat conduction, heat convection, and heat radiation. The total thermal conductivity was related to the solid phase, gas phase, and radiation heat transfer, respectively [24, 73]. There is a large amount of air in the foam, and its thermal conductivity was much lower than that of the Ni/MXene-MF framework. Therefore, high porosity could significantly reduce the thermal conductivity and radiation heat transfer capacity of the solid phase. In addition, the long ligaments of MF could extend the heat conduction path, thereby reducing the heat transfer capacity. Accordingly, Ni/MXene-MF possesses excellent thermal insulation, infrared shielding, and flame retardancy, which could reduce deformation and damage caused in harsh environments, and provide a broad space for infrared stealth applications (Fig. 7e).

\section{Conclusions}

In conclusion, the multifunctional Ni/MXene-MF was successfully prepared through electrostatic self-assembly and dip-coating process. The well-designed Ni/MXene microcapacitor structure and 3D porous structure achieved good impedance matching and strong attenuation efficiency. $\mathrm{Ni} /$ MXene-MF exhibited the best EMA performance with a minimum RL of $-62.7 \mathrm{~dB}$ and the corresponding $\mathrm{EAB}$ reached $6.24 \mathrm{GHz}$ with the thickness of only $2 \mathrm{~mm}$. Meanwhile, the $\mathrm{EAB}$ reached a maximum of $6.88 \mathrm{GHz}$ with a thickness of $1.8 \mathrm{~mm}$. Besides, the novel Ni/MXene-MF exhibits good heat insulation, infrared stealth, and flameretardant functions, making it a huge application potential in harsh environments. Therefore, this work provides design inspiration for multifunctional foams with efficient EM wave absorption properties.

Acknowledgements The authors thank National Natural Science Foundation of China (51803190) and National Key R\&D Program of China (2019YFA0706802) financial support.

Funding Open access funding provided by Shanghai Jiao Tong University.

Open Access This article is licensed under a Creative Commons Attribution 4.0 International License, which permits use, sharing, adaptation, distribution and reproduction in any medium or format, as long as you give appropriate credit to the original author(s) and the source, provide a link to the Creative Commons licence, and indicate if changes were made. The images or other third party material in this article are included in the article's Creative Commons licence, unless indicated otherwise in a credit line to the material. If material is not included in the article's Creative Commons licence and your intended use is not permitted by statutory regulation or exceeds the permitted use, you will need to obtain permission directly from the copyright holder. To view a copy of this licence, visit http://creativecommons.org/licenses/by/4.0/.

Supplementary Information The online version contains supplementary material available at https://doi.org/10.1007/ s40820-022-00812-w.

\section{References}

1. G. Wang, S.J.H. Ong, Y. Zhao, Z.J. Xu, G. Ji, Integrated multifunctional macrostructures for electromagnetic wave absorption and shielding. J. Mater. Chem. A 8(46), 4368-24387 (2020). https://doi.org/10.1039/D0TA08515D 
2. L. Liang, Q. Li, X. Yan, Y. Feng, Y. Wang et al., Multifunctional magnetic $\mathrm{Ti}_{3} \mathrm{C}_{2} \mathrm{~T}_{\mathrm{x}}$ MXene/graphene aerogel with superior electromagnetic wave absorption performance. ACS Nano 15(4), 6622-6632 (2021). https://doi.org/10.1021/acsnano. 0c09982

3. L. Wang, X. Li, X. Shi, M. Huang, X. Li et al., Recent progress of microwave absorption microspheres by magnetic-dielectric synergy. Nanoscale 13(4), 2136-2156 (2021). https://doi.org/ 10.1039/d0nr06267g

4. R. Che, C. Zhi, C. Liang, X. Zhou, Fabrication and microwave absorption of carbon nanotubes $/ \mathrm{CoFe}_{2} \mathrm{O}_{4}$ spinel nanocomposite. Appl. Phys. Lett. 88(3), 033105 (2006). https:// doi.org/10.1063/1.2165276

5. M. Qiao, X. Lei, Y. Ma, L. Tian, X. He et al., Application of yolk-shell $\mathrm{Fe}_{3} \mathrm{O}_{4} @ \mathrm{~N}$-doped carbon nanochains as highly effective microwave-absorption material. Nano Res. 11(3), 1500-1519 (2018). https://doi.org/10.1007/ s12274-017-1767-0

6. R.C. Che, L.M. Peng, X.F. Duan, Q. Chen, X.L. Liang, Microwave absorption enhancement and complex permittivity and permeability of Fe encapsulated within carbon nanotubes. Adv. Mater. 16(5), 401-405 (2004). https://doi. org/10.1002/adma.200306460

7. J. Liu, R. Che, H. Chen, F. Zhang, F. Xia et al., Microwave absorption enhancement of multifunctional composite microspheres with spinel $\mathrm{Fe}_{3} \mathrm{O}_{4}$ cores and anatase $\mathrm{TiO}_{2}$ shells. Small 8(8), 1214-1221 (2012). https://doi.org/10. 1002/smll.201102245

8. Z. Lou, Q. Wang, X. Zhou, U.I. Kara, R.S. Mamtani et al., An angle-insensitive electromagnetic absorber enabling a wideband absorption. J. Mater. Sci. Technol. 113, 33-39 (2022). https://doi.org/10.1016/j.jmst.2021.11.007

9. Q. Liu, Q. Cao, H. Bi, C. Liang, K. Yuan et al., CoNi@ $\mathrm{SiO}_{2} @ \mathrm{TiO}_{2}$ and $\mathrm{CoNi} @ \mathrm{Air} @ \mathrm{TiO}_{2}$ microspheres with strong wideband microwave absorption. Adv. Mater. 28(3), 486-490 (2016). https://doi.org/10.1002/adma.201503149

10. Y. Zhang, Y. Huang, T. Zhang, H. Chang, P. Xiao et al., Broadband and tunable high-performance microwave absorption of an ultralight and highly compressible graphene foam. Adv. Mater. 27(12), 2049-2053 (2015). https://doi. org/10.1002/adma.201405788

11. X. Zhang, S. Li, S. Wang, Z. Yin, J. Zhu et al., Self-supported construction of three-dimensional $\mathrm{MoS}_{2}$ hierarchical nanospheres with tunable high-performance microwave absorption in broadband. J. Phys. Chem. C 120(38), 2201922027 (2016). https://doi.org/10.1021/acs.jpcc.6b06661

12. H. Sun, R. Che, X. You, Y. Jiang, Z. Yang et al., Crossstacking aligned carbon-nanotube films to tune microwave absorption frequencies and increase absorption intensities. Adv. Mater. 26(48), 8120-8125 (2014). https://doi.org/10. 1002/adma.201403735

13. Y. Hou, Y. Yang, C. Deng, C. Li, C. Wang, Implications from broadband microwave absorption of metal-modified SiC fiber mats. ACS Appl. Mater. Interfaces 12(28), 3182331829 (2020). https://doi.org/10.1021/acsami.0c07979
14. M. Cao, Y. Cai, P. He, J. Shu, W. Cao et al., 2D MXenes: electromagnetic property for microwave absorption and electromagnetic interference shielding. Chem. Eng. J. 359, 1265-1302 (2019). https://doi.org/10.1016/j.cej.2018.11. 051

15. X. Li, C. Wen, L. Yang, R. Zhang, X. Li et al., MXene/FeCo films with distinct and tunable electromagnetic wave absorption by morphology control and magnetic anisotropy. Carbon 175, 509-518 (2021). https://doi.org/10.1016/j.carbon.2020. 11.089

16. B. Dai, B. Zhao, X. Xie, T. Su, B. Fan et al., Novel two-dimensional $\mathrm{Ti}_{3} \mathrm{C}_{2} \mathrm{~T}_{\mathrm{x}} \mathrm{MXenes/nano-carbon}$ sphere hybrids for highperformance microwave absorption. J. Mater. Chem. C 6(21), 5690-5697 (2018). https://doi.org/10.1039/c8tc01404c

17. L. Liang, R. Yang, G. Han, Y. Feng, B. Zhao et al., Enhanced electromagnetic wave-absorbing performance of magnetic nanoparticles-anchored 2D $\mathrm{Ti}_{3} \mathrm{C}_{2} \mathrm{~T}_{\mathrm{x}} \mathrm{MXene}$. ACS Appl. Mater. Interfaces 12(2), 2644-2654 (2019). https://doi.org/10.1021/ acsami.9b18504

18. X. Li, W. You, L. Wang, J. Liu, Z. Wu et al., Self-assemblymagnetized MXene avoid dual-agglomeration with enhanced interfaces for strong microwave absorption through a tunable electromagnetic property. ACS Appl. Mater. Interfaces 11(47), 44536-44544 (2019). https://doi.org/10.1021/acsami.9b11861

19. X. Li, X. Yin, C. Song, M. Han, H. Xu et al., Self-assembly core-shell graphene-bridged hollow MXenes spheres 3D foam with ultrahigh specific EM absorption performance. Adv. Funct. Mater. 28(41), 1803938 (2018). https://doi.org/10.1002/ adfm.201803938

20. J. Yan, Y. Huang, Y. Yan, X. Zhao, P. Liu, The composition design of mof-derived co-fe bimetallic autocatalysis carbon nanotubes with controllable electromagnetic properties. Compos. Part A Appl. Sci. Manuf. 139, 106107 (2020). https://doi. org/10.1016/j.compositesa.2020.106107

21. Y. Cui, Z. Liu, Y. Zhang, P. Liu, M. Ahmad et al., Wrinkled three-dimensional porous MXene/Ni composite microspheres for efficient broadband microwave absorption. Carbon 181, 58-68 (2021). https://doi.org/10.1016/j.carbon.2021.05.022

22. A. Sheng, Y. Yang, D. Yan, K. Dai, H. Duan et al., Self-assembled reduced graphene oxide/nickel nanofibers with hierarchical core-shell structure for enhanced electromagnetic wave absorption. Carbon 167, 530-540 (2020). https://doi.org/10. 1016/j.carbon.2020.05.107

23. X. Zhu, Y. Dong, Z. Xiang, L. Cai, F. Pan et al., Morphologycontrollable synthesis of polyurethane-derived highly crosslinked 3D networks for multifunctional and efficient electromagnetic wave absorption. Carbon 182, 254-264 (2021). https://doi.org/10.1016/j.carbon.2021.06.028

24. W. Gu, J. Tan, J. Chen, Z. Zhang, Y. Zhao et al., Multifunctional bulk hybrid foam for infrared stealth, thermal insulation, and microwave absorption. ACS Appl. Mater. Interfaces 12(25), 28727-28737 (2020). https://doi.org/10.1021/acsami. 0c09202

25. K. Su, Y. Wang, K. Hu, X. Fang, J. Yao et al., Ultralight and high-strength $\mathrm{SiC}_{\mathrm{nw}} @ \mathrm{SiC}$ foam with highly efficient microwave absorption and heat insulation properties. ACS Appl. 
Mater. Interfaces 13(18), 22017-22030 (2021). https://doi.org/ 10.1021/acsami.1c03543

26. X. Yan, X. Huang, Y. Chen, Y. Liu, L. Xia et al., A theoretical strategy of pure carbon materials for lightweight and excellent absorption performance. Carbon 174, 662-672 (2021). https:// doi.org/10.1016/j.carbon.2020.11.044

27. Z. Xiang, X. Zhu, Y. Dong, X. Zhang, Y. Shi et al., Enhanced electromagnetic wave absorption of magnetic Co nanoparticles/CNTs/EG porous composites with waterproof, flameretardant and thermal management functions. J. Mater. Chem. A 9(32), 17538-17552 (2021). https://doi.org/10.1039/d1ta0 $5181 \mathrm{~d}$

28. X. Zhang, Z. Liu, B. Deng, L. Cai, Y. Dong et al., Honeycomb-like $\mathrm{NiCo}_{2} \mathrm{O}_{4} @ \mathrm{M}_{\mathrm{n}} \mathrm{O}_{2}$ nanosheets array/3D porous expanded graphite hybrids for high-performance microwave absorber with hydrophobic and flame-retardant functions. Chem. Eng. J. 419, 129547 (2021). https://doi.org/10.1016/j. cej.2021.129547

29. Z. Zhang, J. Tan, W. Gu, H. Zhao, J. Zheng et al., Cellulosechitosan framework/polyailine hybrid aerogel toward thermal insulation and microwave absorbing application. Chem. Eng. J. 395, 125190 (2020). https://doi.org/10.1016/j.cej. 2020.125190

30. Y. Dong, X. Zhu, F. Pan, Z. Xiang, X. Zhang et al., Fireretardant and thermal insulating honeycomb-like $\mathrm{NiS}_{2} / \mathrm{SnS}_{2}$ nanosheets@3D porous carbon hybrids for high-efficiency electromagnetic wave absorption. Chem. Eng. J. 426, 131272 (2021). https://doi.org/10.1016/j.cej.2021.131272

31. W. Gu, J. Sheng, Q. Huang, G. Wang, J. Chen et al., Environmentally friendly and multifunctional shaddock peelbased carbon aerogel for thermal-insulation and microwave absorption. Nano-Micro Lett. 13, 102 (2021). https://doi. org/10.1007/s40820-021-00635-1

32. B. Deng, Z. Liu, F. Pan, Z. Xiang, X. Zhang et al., Electrostatically self-assembled two-dimensional magnetized MXene/hollow $\mathrm{Fe}_{3} \mathrm{O}_{4}$ nanoparticle hybrids with high electromagnetic absorption performance and improved impendence matching. J. Mater. Chem. A 9(6), 3500-3510 (2021). https://doi.org/10.1039/d0ta10551a

33. M. Alhabeb, K. Maleski, B. Anasori, P. Lelyukh, L. Clark et al., Guidelines for synthesis and processing of twodimensional titanium carbide $\left(\mathrm{Ti}_{3} \mathrm{C}_{2} \mathrm{~T}_{\mathrm{x}}\right.$ MXene). Chem. Mat. 29(18), 7633-7644 (2017). https://doi.org/10.1021/ acs.chemmater.7b02847

34. L. Liang, G. Han, Y. Li, B. Zhao, B. Zhou et al., Promising $\mathrm{Ti}_{3} \mathrm{C}_{2} \mathrm{~T}_{\mathrm{x}} \mathrm{MXene} / \mathrm{Ni}$ chain hybrid with excellent electromagnetic wave absorption and shielding capacity. ACS Appl. Mater. Interfaces 11(28), 25399-25409 (2019). https://doi. org/10.1021/acsami.9b07294

35. P. He, M.S. Cao, J.C. Shu, Y.Z. Cai, X.X. Wang et al., Atomic layer tailoring titanium carbide MXene to tune transport and polarization for utilization of electromagnetic energy beyond solar and chemical energy. ACS Appl. Mater. Interfaces 11(13), 12535-12543 (2019). https://doi.org/10. 1021/acsami.9b00593
36. X. Li, M. Zhang, W. You, K. Pei, Q. Zeng et al., Magnetized MXene microspheres with multiscale magnetic coupling and enhanced polarized interfaces for distinct microwave absorption via a spray-drying method. ACS Appl. Mater. Interfaces 12(15), 18138-18147 (2020). https://doi.org/10. 1021/acsami.0c00935

37. R. Sun, H.B. Zhang, J. Liu, X. Xie, R. Yang et al., Highly conductive transition metal carbide/carbonitride (MXene)@ polystyrene nanocomposites fabricated by electrostatic assembly for highly efficient electromagnetic interference shielding. Adv. Funct. Mater. 27(45), 1702807 (2017). https://doi.org/10.1002/adfm.201702807

38. L. Liang, C. Yao, X. Yan, Y. Feng, X. Hao et al., High-efficiency electromagnetic interference shielding capability of magnetic $\mathrm{Ti}_{3} \mathrm{C}_{2} \mathrm{~T}_{\mathrm{x}}$ MXene/CNT composite film. J. Mater. Chem. A 9(43), 24560-24570 (2021). https://doi.org/10.1039/ d1ta07781c

39. J. Wang, L. Liu, S. Jiao, K. Ma, J. Lv et al., Hierarchical carbon Fiber@MXene@ $\mathrm{MoS}_{2}$ core-sheath synergistic microstructure for tunable and efficient microwave absorption. Adv. Funct. Mater. 30(45), 2002595 (2020). https://doi.org/10.1002/ adfm.202002595

40. Z. Wu, K. Pei, L. Xing, X. Yu, W. You et al., Enhanced microwave absorption performance from magnetic coupling of magnetic nanoparticles suspended within hierarchically tubular composite. Adv. Funct. Mater. 29(28), 1901448 (2019). https://doi.org/10.1002/adfm.201901448

41. F. Ye, Q. Song, Z. Zhang, W. Li, S. Zhang et al., Direct growth of edge-rich graphene with tunable dielectric properties in porous $\mathrm{Si}_{3} \mathrm{~N}_{4}$ ceramic for broadband high-performance microwave absorption. Adv. Funct. Mater. 28(17), 1707205 (2018). https://doi.org/10.1002/adfm.201707205

42. D. Liu, Y. Du, Z. Li, Y. Wang, P. Xu et al., Facile synthesis of 3D flower-like Ni microspheres with enhanced microwave absorption properties. J. Mater. Chem. C 6(36), 9615-9623 (2018). https://doi.org/10.1039/C8TC02931H

43. G. Liu, J. Tu, C. Wu, Y. Fu, C. Chu et al., High-yield twodimensional metal-organic framework derivatives for wideband electromagnetic wave absorption. ACS Appl. Mater. Interfaces 13(17), 20459-20466 (2021). https://doi.org/10. 1021/acsami.1c00281

44. C. Xu, L. Wang, X. Li, X. Qian, Z. Wu et al., Hierarchical magnetic network constructed by cofe nanoparticles suspended within "tubes on rods" matrix toward enhanced microwave absorption. Nano-Micro Lett. 13, 47 (2021). https://doi. org/10.1007/s40820-020-00572-5

45. X. Zhu, Y. Dong, F. Pan, Z. Xiang, Z. Liu et al., Covalent organic framework-derived hollow core-shell $\mathrm{Fe} / \mathrm{Fe}_{3} \mathrm{O}_{4} @$ porous carbon composites with corrosion resistance for lightweight and efficient microwave absorption. Compos. Commun. 25, 100731 (2021). https://doi.org/10.1016/j.coco.2021. 100731

46. M. Zhou, W. Gu, G. Wang, J. Zheng, C. Pei et al., Sustainable wood-based composites for microwave absorption and electromagnetic interference shielding. J. Mater. Chem. A 8(46), 24267-24283 (2020). https://doi.org/10.1039/d0ta08372k 
47. L. Liang, G. Song, Z. Liu, J. Chen, L. Xie et al., Constructing $\mathrm{Ni}_{12} \mathrm{P}_{5} / \mathrm{Ni}_{2} \mathrm{P}$ heterostructures to boost interfacial polarization for enhanced microwave absorption performance. ACS Appl. Mater. Interfaces 12(46), 52208-52220 (2020). https://doi.org/ 10.1021/acsami.0c16287

48. F. Pan, Z. Liu, B. Deng, Y. Dong, X. Zhu et al., Lotus leafderived gradient hierarchical porous $\mathrm{C} / \mathrm{MoS}_{2}$ morphology genetic composites with wideband and tunable electromagnetic absorption performance. Nano-Micro Lett. 13, 43 (2021). https://doi.org/10.1007/s40820-020-00568-1

49. X. Zhang, X. Wang, P. Sha, B. Wang, Y. Ding et al., Highefficiency electromagnetic wave absorption of epoxy composites filled with ultralow content of reduced graphene/ carbon nanotube oxides. Compos. Sci. Technol. 189, 108020 (2020). https://doi.org/10.1016/j.compscitech.2020.108020

50. G. He, Y. Duan, H. Pang, Microwave absorption of crystalline Fe/MnO@C nanocapsules embedded in amorphous carbon. Nano-Micro Lett. 12, 57 (2020). https://doi.org/10. 1007/s40820-020-0388-4

51. B. Zhao, Y. Li, Q. Zeng, L. Wang, J. Ding et al., Galvanic replacement reaction involving core-shell magnetic chains and orientation-tunable microwave absorption properties. Small 16(40), 2003502 (2020). https://doi.org/10.1002/smll. 202003502

52. H. Ji, J. Li, J. Zhang, Y. Yan, Remarkable microwave absorption performance of ultralight graphene-polyethylene glycol composite aerogels with a very low loading ratio of graphene. Compos. Part A Appl. Sci. Manuf. 123, 158-169 (2019). https://doi.org/10.1016/j.compositesa.2019.05.012

53. B. Wen, M. Cao, M. Lu, W. Cao, H. Shi et al., Reduced graphene oxides: light-weight and high-efficiency electromagnetic interference shielding at elevated temperatures. Adv. Mater. 26(21), 3484-3489 (2014). https://doi.org/10.1002/ adma.201400108

54. R. Wang, M. He, Y. Zhou, S. Nie, Y. Wang et al., Selfassembled 3D flower-like composites of heterobimetallic phosphides and carbon for temperature-tailored electromagnetic wave absorption. ACS Appl. Mater. Interfaces 11(41), 38361-38371 (2019). https://doi.org/10.1021/acsami.9b148 73

55. Y. Guo, D. Wang, T. Bai, H. Liu, Y. Zheng et al., Electrostatic self-assembled $\mathrm{NiFe}_{2} \mathrm{O}_{4} / \mathrm{Ti}_{3} \mathrm{C}_{2} \mathrm{~T}_{\mathrm{x}}$ MXene nanocomposites for efficient electromagnetic wave absorption at ultralow loading level. Adv. Compos. Hybrid Mater. 4, 602-613 (2021). https://doi.org/10.1007/s42114-021-00279-0

56. H. Peng, M. He, Y. Zhou, Z. Song, Y. Wang et al., Low-temperature carbonized biomimetic cellulose nanofiber/MXene composite membrane with excellent microwave absorption performance and tunable absorption bands. Chem. Eng. J. (2021). https://doi.org/10.1016/j.cej.2021.133269

57. C. Wen, X. Li, R. Zhang, C. Xu, W. You et al., High-density anisotropy magnetism enhanced microwave absorption performance in $\mathrm{Ti}_{3} \mathrm{C}_{2} \mathrm{~T}_{\mathrm{x}} \mathrm{MXene} @ \mathrm{Ni}$ microspheres. ACS Nano (2021). https://doi.org/10.1021/acsnano.1c08957

58. B. Zhao, G. Shao, B. Fan, B. Sun, K. Guan et al., Facile synthesis and novel microwave electromagnetic properties of flower-like Ni structures by a solvothermal method. J. Mater. Sci. Mater. Electron. 25(8), 3614-3621 (2014). https://doi. org/10.1007/s10854-014-2064-2

59. Z. Xu, M. He, Y. Zhou, M. Zhang, S. Feng et al., Rime-like carbon paper@ $\mathrm{Bi}_{2} \mathrm{~S}_{3}$ hybrid structure for efficient and broadband microwave absorption. Chem. Eng. J. 428, 131127 (2022). https://doi.org/10.1016/j.cej.2021.131127

60. P. Xie, Y. Liu, M. Feng, M. Niu, C. Liu et al., Hierarchically porous $\mathrm{Co} / \mathrm{C}$ nanocomposites for ultralight highperformance microwave absorption. Adv. Compos. Hybrid Mater. 4, 173-185 (2021). https://doi.org/10.1007/ s42114-020-00202-Z

61. Z. Zhang, Y. Zhao, Z. Li, L. Zhang, Z. Liu et al., Synthesis of carbon $/ \mathrm{SiO}_{2}$ core-sheath nanofibers with Co-Fe nanoparticles embedded in via electrospinning for high-performance microwave absorption. Adv. Compos. Hybrid Mater. (2021). https://doi.org/10.1007/s42114-021-00350-w

62. P. Zhang, X. Zhang, B. Li, L. Xu, F. Dang et al., Enhanced microwave absorption performance in an ultralight porous single-atom Co-N-C absorber. Adv. Compos. Hybrid Mater. 4, 1292-1301 (2021). https://doi.org/10.1007/ s42114-021-00308-y

63. G. Qi, Y. Liu, L. Chen, P. Xie, D. Pan et al., Lightweight $\mathrm{Fe}_{3} \mathrm{C} @ \mathrm{Fe} / \mathrm{C}$ nanocomposites derived from wasted cornstalks with high-efficiency microwave absorption and ultrathin thickness. Adv. Compos. Hybrid Mater. 4, 1226-1238 (2021). https://doi.org/10.1007/s42114-021-00368-0

64. N. Wu, B. Zhao, J. Liu, Y. Li, Y. Chen et al., MOF-derived porous hollow $\mathrm{Ni} / \mathrm{C}$ composites with optimized impedance matching as lightweight microwave absorption materials. Adv. Compos. Hybrid Mater. 4, 707-715 (2021). https://doi. org/10.1007/s42114-021-00307-z

65. P. Hu, S. Dong, F. Yuan, X. Li, C. Hong, Hollow carbon microspheres modified with $\mathrm{NiCo}_{2} \mathrm{~S}_{4}$ nanosheets as a highperformance microwave absorber. Adv. Compos. Hybrid Mater. (2021). https://doi.org/10.1007/s42114-021-00318-w

66. Y. Li, Y. Qing, W. Li, M. Zong, F. Luo, Novel Magnéli $\mathrm{Ti}_{4} \mathrm{O}_{7} / \mathrm{Ni} /$ poly (vinylidene fluoride) hybrids for high-performance electromagnetic wave absorption. Adv. Compos. Hybrid Mater. 4, 1027-1038 (2021). https://doi.org/10.1007/ s42114-021-00297-y

67. F. Luo, D. Liu, T. Cao, H. Cheng, J. Kuang et al., Study on broadband microwave absorbing performance of gradient porous structure. Adv. Compos. Hybrid Mater. 4, 591-601 (2021). https://doi.org/10.1007/s42114-021-00275-4

68. H.G. Shi, H.B. Zhao, B.W. Liu, Y.Z. Wang, Multifunctional flame-retardant melamine-based hybrid foam for infrared stealth, thermal insulation, and electromagnetic interference shielding. ACS Appl. Mater. Interfaces 13, 26505-26514 (2021). https://doi.org/10.1021/acsami.1c07363

69. Y. Li, X. Liu, X. Nie, W. Yang, Y. Wang et al., Multifunctional organic-inorganic hybrid aerogel for self-cleaning, heat-insulating, and highly efficient microwave absorbing material. Adv. Funct. Mater. 29(10), 1807624 (2019). https://doi.org/10.1002/adfm.201807624 
70. B. Du, D. Zhang, J. Qian, M. Cai, C. He et al., Multifunctional carbon nanofiber-sic nanowire aerogel films with superior microwave absorbing performance. Adv. Compos. Hybrid Mater. 4, 1281-1291 (2021). https://doi.org/10.1007/ s42114-021-00286-1

71. H. Cheng, Y. Pan, Q. Chen, R. Che, G. Zheng et al., Ultrathin flexible poly(vinylidene fluoride)/MXenes/silver nanowires film with outstanding specific EMI shielding and high heat dissipation. Adv. Compos. Hybrid Mater. 4, 505-513 (2021). https://doi.org/10.1007/s42114-021-00224-1
72. Q. Gao, Y. Pan, G. Zheng, C. Liu, C. Shen et al., Flexible multilayered MXene/thermoplastic polyurethane films with excellent electromagnetic interference shielding, thermal conductivity and management performances. Adv. Compos. Hybrid Mater. 4, 274-285 (2021). https://doi.org/10.1007/ s42114-021-00221-4

73. A.A. Gunay, H. Kim, N. Nagarajan, M. Lopez, R. Kantharaj et al., Optically transparent thermally insulating silica aerogels for solar thermal insulation. ACS Appl. Mater. Interfaces 10(15), 12603-12611 (2018). https://doi.org/10.1021/acsami. $7 \mathrm{~b} 18856$ 